\title{
CONNECTEDNESS IN THE SCALE OF A UNIFORM SPACE
}

\author{
G. C. LESLIE and D. C. KENT \\ (Received 8 September 1969) \\ Communicated by B. Mond
}

Associated with each uniform space $(S, \mathscr{U})$ there is a uniform lattice $(P, \mathscr{V}, \leqq)$ called the scale of $(S, \mathscr{U})$. The scale was first introduced by D. Bushaw (see [2] and [3]) for the purpose of studying stability in topological dynamics. Further properties of the scale were investigated in [4], and this paper pursues one of the research problems suggested in the latter paper.

We show that $(P, \mathscr{V})$ is uniformly connected if and only if $(S, \mathscr{U})$ is bounded. Sufficient conditions are found on $(S, \mathscr{U})$ which make the scale connected and arcwise connected, and the relationship between connectedness and local connectedness is also considered. The concluding section characterizes total disconnectedness in the scale, which turns out to be equivalent to discreteness in the original space.

Starting with a uniform space $(S, \mathscr{U})$, let $\alpha$ be a non-empty anti-residual subcollection of the uniformity $\mathscr{U}$; that is, if $U \in \alpha, V \in \mathscr{U}$, and $V \supset U$, then $V \in \alpha$. Such sets $\alpha$ are called prefilters relative to $(S, \mathscr{U})$, and the set of all prefilters for $(S, \mathscr{U})$, equipped with an order relation described below, is called the scale of $(S, \mathscr{U})$. We also use the term scale ambiguously to describe the uniform space $(P, \mathscr{V})$, where $\mathscr{V}$ is the uniformity for $P$ generated by sets of the form $U^{\prime}=\{(\alpha, \beta) \in P \times P: A \in \alpha$ implies $U A \in \beta$ and $B \in \beta$ implies $U B \in \alpha\}$, for all $U \in \mathscr{U}$. That $\left\{U^{\prime}: U \in \mathscr{U}\right\}$ is the base for a uniformity on $P$ follows immediately from the fact that, for each $U \in \mathscr{U}, U^{\prime 2}=U^{2 \prime}$ (Proposition 2, [4]). The scale is always a complete uniform space, but is compact if and only if the original space is either finite or discrete [4].

The scale forms a complete distributive lattice under the ordering: $\alpha \leqq \beta$ whenever $\alpha \supset \beta$; the lattice operations are uniformly continuous (Theorem 1, [4]). The least element of $P$ is $\mathscr{U}$, denoted $O$; the greatest element is $\{S \times S\}$, denoted $l$.

A uniform space $(S, \mathscr{U})$ is said to be bounded if, for each $U \in \mathscr{U}$, there is a positive integer $n$ such that $U^{n}=S \times S$. In this usage (which is nonstandard) we follow [1], and it is worth noting that boundedness neither implies nor is implied 
by total boundedness, although a connected totally bounded space is bounded.

A uniform space $(S, \mathscr{U})$ is said to be uniformly connected if every uniformly continuous map of the space into a discrete space is a constant map. The following lemma, established in [5], shows that boundedness implies uniform connectedness; Theorem 1 shows that for scales these concepts are equivalent.

Lemma 1. (Mrówka and Pervin). A uniform space ( $S, \mathscr{U}$ ) is uniformly connected if and only if, for each pair $x, y$ of elements in $S$ and each $U \in \mathscr{U}$, there is an integer $n$ such that $(x, y) \in U^{n}$.

THEOREM 1. The following statements are equivalent.

(1) $(S, \mathscr{U})$ is bounded.

(2) $(P, \mathscr{V})$ is bounded.

(3) $(P, \mathscr{V})$ is uniformly connected.

Proof. That (1) implies (2) follows from Proposition 2, [4]. That (2) implies (3) is an easy consequence of Lemma 1. To show that (3) implies (1), assume that $(P, \mathscr{V})$ is uniformly connected and let $U \in \mathscr{U}$. By Lemma 1 , there is an integer $n$ such that $(O, l) \in U^{\prime n}$; furthermore we can assume by Proposition 2, [4] that $U^{\prime n}=U^{n \prime}$. Thus for each $V \in \mathscr{U}, U^{n} V=S \times S$, and in particular $U^{n+1}=S \times S$. But this is precisely what we need to establish that $(S, \mathscr{U})$ is bounded, and so the proof is complete.

A topological space is connected if and only if the only mapping of the space into a discrete space is a constant mapping. Thus connectedness implies uniform connectedness in a uniform space, and boundedness in $(S, \mathscr{U})$ is necessary for connectedness in $(P, \mathscr{V})$.

Let $R$ be the equivalence relation on $P$ defined by: $(\alpha, \beta) \in R$ whenever $(\alpha, \beta) \in U^{\prime}$, all $U \in \mathscr{U}$. Let $\alpha_{R}$ denote the $R$-equivalence class containing $\alpha$; we have from [4] that

where

$$
\alpha_{R}=\left\{\beta: \alpha_{0} \leqq \beta \leqq \alpha^{0}\right\}
$$

and

$$
\alpha_{0}=\{U \in \mathscr{U}: V U \in \alpha, \text { all } V \in \mathscr{U}\}
$$

$$
\alpha^{0}=\{U \in \mathscr{U}: V A \subset U \text { for some } V \in \mathscr{U} \text { and some } A \in \alpha\} .
$$

The following proposition is trivial.

Proposition 1. Each R-equivalence class is a connected set in $(P, \mathscr{V})$.

It will be convenient to introduce the following closed interval notation: for $\alpha \leqq \beta$, let $[\alpha, \beta]=\{\gamma \in P: \alpha \leqq \gamma \leqq \beta\}$. For each $U \in \mathscr{U}$ and $\alpha \in P$, let

$$
\begin{aligned}
& \alpha_{U}=\{V \in \mathscr{U}: U V \in \alpha\}, \\
& \alpha^{U}=\{V \in \mathscr{U}: \text { for some } A \in \alpha, U A \subset V\} .
\end{aligned}
$$


It is a simple matter to show that $U^{\prime}[\alpha]=\left[\alpha_{U}, \alpha^{U}\right]$, and this immediately yields the next proposition.

Proposition 2. The collection $\left\{\left[\alpha_{U}, \alpha^{U}\right]: U \in \mathscr{U}\right\}$ forms a basic system of neighborhoods at $\alpha$ for the uniform topology on $P$ generated by $\mathscr{V}$.

In case $\alpha=O$, note that $O_{U}=O$. Let $\langle U\rangle$ denote the prefilter consisting of all oversets of $U$. Since $O^{V} \leqq\langle U\rangle \leqq O^{U}$ for $V^{2} \subset U$, it follows from Proposition 2 that the collection $\{[O,\langle U\rangle]: U \in \mathscr{U}\}$ is a basic neighborhood system for $O$. This fact will be used in several subsequent proofs.

Given prefilters $\alpha, \beta$ in $P$, we define their product as follows:

$$
\alpha \beta=\{W \in \mathscr{U}: \text { for some } U \in \alpha \text { and } V \in \beta, W \supset U V\} ;
$$

for a subset $M$ of $P$, let $M \alpha=\{\beta \alpha: \beta \in M\}$. It can be easily verified that the mapping $\alpha^{\prime}$ of $P$ into itself which carries each element $\beta$ into $\beta \alpha$ is uniformly continuous. Let $M \vee \alpha=\{\beta \vee \alpha: \beta \in M\}$ and $M \wedge \alpha=\{\beta \wedge \alpha: \beta \in M\}$. The lattice operations $\vee$ and $\wedge$ in $P$ are uniformly continuous (Theorem 1, [4]), and thus we obtain the following result.

Proposition 3. If $M$ is a connected (arcwise connected) subset of $P$, then the sets $M \propto, M \vee \alpha$, and $M \wedge \alpha$ are likewise connected (arcwise connected) for any choice of $\alpha$.

Proposition 4. If there is a connected (arcwise connected) subset $C$ of $P$ containing $\alpha$ and $\beta$, then $[\alpha \wedge \beta, \alpha \vee \beta]$ is connected (arcwise connected).

Proof. The sets $C \vee \alpha$ and $C \wedge \alpha$ are connected sets which contain $\alpha$, and hence $A=(C \vee \alpha) \cup(C \wedge \alpha)$ is a connected set which contains both $\alpha \vee \beta$ and $\alpha \wedge \beta$. If $\gamma \in[\alpha \wedge \beta, \alpha \vee \beta], A \vee \gamma$ is a connected set which contains $\gamma$ and $\alpha \vee \beta$. Thus

$$
B=\cup\{A \vee \gamma: \gamma \in[\alpha \wedge \beta, \alpha \vee \beta]\}
$$

is a connected set which includes $[\alpha \wedge \beta, \alpha \vee \beta]$ as a subset. But

$$
[\alpha \wedge \beta, \alpha \vee \beta]=(B \vee(\alpha \wedge \beta)) \wedge(\alpha \vee \beta),
$$

and the proof is complete.

COROLLARY 1. The scale of a uniform space is connected (arcwise connected) if $O$ and l belong to the same connected (arcwise connected) component.

CoROllary 2. If the closed interval $[\alpha, \beta]$ in $P$ is a subset of a connected (arcwise connecled) set $M$, then $[\alpha, \beta]$ is connected (arcwise connected).

COROLLARY 3. The scale of a uniform space is locally connected (locally arcwise connected) if and only if there is a connected (arcwise connected) neighborhood for each point.

The third corollary follows from Corollary 2 and Proposition 2. 
THEOREM 2. The scale $(P, \mathscr{V})$ of a uniform space $(S, \mathscr{U})$ is locally connected (locally arcwise connected) if and only if there is a connected (arcwise connected) neighborhood of $O$.

Proof. Let $N$ be a connected neighborhood of $O$; then $N$ contains a basic neighborhood of the form $[O,\langle U\rangle]$, and the latter is connected by Corollary 2 . We will show that the set $\left[\alpha_{U}, \alpha^{U}\right]$ is connected for any $\alpha \in P$, and the desired result will then follow by Proposition 2 and Corollary 3 .

Let $A=[O,\langle U\rangle] \alpha$ and let $B=[O,\langle U\rangle] \alpha_{U} \cdot A$ contains the points $\alpha^{0}=O \alpha$ and $\alpha^{U}=\langle U\rangle \alpha$; thus, by Propositions 1,3 , and $4,\left[\alpha, \alpha^{U}\right]$ is connected. $B$ contains the points $\left(\alpha_{U}\right)^{0}$ and $\left(\alpha_{U}\right)^{U}$, and by similar reasoning $\left[\alpha_{U},\left(\alpha_{U}\right)^{U}\right]$ is connected. But $\alpha$ is a member of each of the indicated closed intervals, and a third application of Proposition 4 yields the connectedness of $\left[\alpha_{U}, \alpha^{U}\right]$, thus completing the proof.

THEOREM 3. The scale $(P, \mathscr{V})$ of a bounded uniform space $(S, \mathscr{U})$ is connected (arcwise connected) if and only if it is locally connected (locally arcwise connected).

Proof. That connectedness implies local connectedness follows from Theorem 1 and Corollary 2. Assume that $(S, \mathscr{U})$ is bounded and that $(P, \mathscr{V})$ is locally connected; choose a connected neighborhood for $O$ of the form $N=[O,\langle U\rangle]$. Let $n$ be an integer such that $U^{n}=S \times S$. Then the set

$$
N \cup\langle U\rangle_{R} \cup N\langle U\rangle \cup\left\langle U_{2}\right\rangle_{R} \cup N\left\langle U^{2}\right\rangle \cup \cdots \cup N\left\langle U^{n-1}\right\rangle
$$

is a connected set which contains both $O$ and $l$, and $(P, \mathscr{V})$ is connected by Corollary 1 .

Boundedness in $(S, \mathscr{U})$ is not a necessary condition for local connectedness in $(P, \mathscr{V})$ as we shall see in the next section (Theorem 5). In the next proposition, we find a similar but weaker condition which is necessary for local connectedness in the scale, and from this result one can construct examples to show that the scale of a uniform space is not always locally connected.

PROPOSITION 5. If $(P, \mathscr{V})$ is locally connected, then there is an entourage $U$ in $\mathscr{U}$ such that, for every $V \in \mathscr{U}, V^{n} \subset U$ for some integer $n$.

Proof. Assume that the given condition on $\mathscr{U}$ fails; thus, given $U$ in $\mathscr{U}$, there is $V \in \mathscr{U}$ such that $V^{n}$ is not a subset of $U$ for all integers $n$. Let $J=\{\alpha \in P$ : $V^{n} \in \alpha$ for some $\left.n\right\}$ and $K=\left\{\alpha \in P: V^{n} \notin \alpha\right.$ for all $\left.n\right\}$. It can be shown without difficulty that $J$ and $K$ are both open sets. But $O \in J$ and $\langle U\rangle \in K$; thus the neighborhood $[O,\langle U\rangle]$ of $O$ is not connected. But $U$ is arbitrary, and so $O$ has no connected neighborhood.

We will now give conditions on $(S, \mathscr{U})$ which are sufficient for connectedness, local connectedness, and arcwise connectedness in $(P, \mathscr{V})$. 
A uniform space satisfies Condition $A$ if there is a base $\mathscr{B}$ for the uniformity which is closed under unions and finite compositions such that, given a chain $\left\{V_{\lambda}\right\}$ in $\mathscr{B}$ and $W \in \mathscr{U}$, there is an index $\lambda$ such that $W V_{\lambda} \supset \cup\left\{V_{\lambda}\right\}$.

Let $(S, d)$ be a pseudo-metric space, and for each $\varepsilon>0$ let $U_{\varepsilon}$ be the entourage $\{(x, y): d(x, y)<\varepsilon\}$. If $U_{\varepsilon} U_{\delta}=U_{\varepsilon+\delta}$ for all positive real numbers $\varepsilon$, $\delta$ then $(S, d)$ is said to satisfy Condition $B$.

The uniformity derived from a pseudo-metric which satisfies Condition B will surely satisfy Condition $A$, since $\mathscr{B}$ can be chosen to consist of $\left\{U_{\varepsilon}: \varepsilon>0\right\}$.

THEOREM 4. If $(S, \mathscr{U})$ is a bounded uniform space which satisfies Condition A, then $(P, \mathscr{V})$ is connected.

Proof. Assume that there is a subset $M$ of $P$ which is both open and closed, distinct from $P$, and contains an element $\alpha$. We shall show that $l$ is in $M$. If $N$ is the complement of $M$, then precisely the same argument establishes that $l \in N$, a contradiction.

Let $\mathscr{S}=\left\{V \in \mathscr{U}: \alpha^{V} \in M, V \in \mathscr{B}\right\}$, where $\mathscr{B}$ is the base for $\mathscr{U}$ specified in Condition A. $\mathscr{S}$ is non-empty since $M$ is open. We shall partially order $\mathscr{S}$ by set inclusion and apply Zorn's Lemma to establish the existence of a maximal element $U$ in $\mathscr{S}$. Indeed, if $\left\{V_{i}\right\}$ is a chain in $\mathscr{S}$, and $V=\cup\left\{V_{i}\right\}$, then Condition A is precisely what is needed to show that the net $\left\{\alpha^{V_{i}}\right\}$ converges to $\alpha^{V}$. Since $M$ is closed, $\alpha^{V}$ is in $M$, and $V \in \mathscr{S}$.

Since $\alpha^{U} \in M$ and $M$ is open, there is $W \in \mathscr{B}$ such that $\left(\alpha^{U}\right)^{W}=\alpha^{W U} \in M$. Thus $W U \in \mathscr{S}$ (recall that $\mathscr{S}$ is closed under finite compositions) and so $W U=U$. The proof will be completed by showing that, necessarily, $U=S \times S$. Indeed, $W^{2} U=W W U=W U=U$, and by induction $W^{k} U=U$ for all integers $k$. But $(S, \mathscr{U})$ is bounded, and so $W^{n}=S \times S$ for some integer $n$. Thus $U=S \times S$, and $\alpha^{U}=l \in M$, which completes the proof.

A pseudo-metric space $(S, d)$ is bounded if there is an upper bound for the function $d$. A bounded pseudo-metric always induces a bounded uniformity, but the converse is false in general (see [1]). If $(S, d)$ satisfies Condition $\mathrm{B}$, however, then boundedness in $(S, d)$ is equivalent to boundedness in the associated uniform space, and hence this term as used in the next theorem can be interpreted in either sense.

TheоReм 5. If a pseudo-metric space $(S, d)$ satisfies Condition $B$, then the scale $(P, \mathscr{V})$ of the associated uniform space is locally arcwise connected. If, in addition, $(S, d)$ is bounded, then $(P, \mathscr{V})$ is arcwise connected.

Proof. We can assume with no loss of generality that $d$ assumes values greater than or equal to 1 . Let $f$ be the function mapping the unit interval $[0,1]$ into $(P, \mathscr{V})$ defined by $f(r)=\left\langle U_{r}\right\rangle$, where $\left\langle U_{r}\right\rangle$ denotes the prefilter consisting of all oversets of $U_{r}$; in particular let $f(0)=O$. Condition B guarantees that $f$ is uniformly continuous. Thus, by Proposition 4, the interval $\left[O,\left\langle U_{1}\right\rangle\right]$ is arcwise 
connected, and it follows from Theorem 2 that $(P, \mathscr{V})$ is locally arcwise connected. If $(S, d)$ is also bounded, then $(P, \mathscr{V})$ is arcwise connected by Theorem 3 .

It can be shown that if $f$ is a uniformly continuous mapping from $\left(S_{1}, \mathscr{U}_{1}\right)$ onto $\left(S_{2}, \mathscr{U}_{2}\right)$, then $f$ induces in a natural way a uniformly continuous mapping from the scale of $\left(S_{1}, \mathscr{U}_{1}\right)$ onto the scale of $\left(S_{2}, \mathscr{U}_{2}\right)$. Thus any space which is the uniformly continuous image of a space satisfying the conditions of Theorem 3 (Theorem 4) has a connected (arcwise connected) scale.

The retracted scale $\left(P_{R}, \mathscr{V}_{R}\right)$ of a uniform space $(S, \mathscr{U})$ is constructed by taking $P_{R}$ to be the set of all $R$-equivalence classes and imposing the quotient uniformity derived from $(P, \mathscr{V})$ (see [4]). It can be shown that the retracted scale is connected (resp. uniformly connected, locally connected, arcwise connected, locally arcwise connected) when and only when the scale is connected (resp. uniformly connected, locally connected, arcwise connected, locally arcwise connected).

The following three propositions lead to a very thorough characterization of total disconnectedness in the scale $(P, \mathscr{V})$ of a non-trivial uniform space $(S, \mathscr{U})$.

Proposition 6. $(P, \mathscr{V})$ is Hausdorff if and only if, for each $U \in \mathscr{U}$, there exists $V \in \mathscr{U}$ such that $V U=U$.

Proof. Let $(P, \mathscr{V})$ be Hausdorff and $U \in \mathscr{U}$ such that $U \neq S \times S$. Let $\alpha=\langle U\rangle$ and $\beta=\alpha-\{U\}$. Since $(P, \mathscr{V})$ is Hausdorff, $\alpha$ and $\beta$ are not $R$-related, and thus there must be an entourage $V$ in $\mathscr{U}$ such that $V U \notin \beta$. But since $U \subset V U$, this implies that $V U=U$. Conversely, assume the given condition, and assume that $(\alpha, \beta) \in U^{\prime}$ for all $U \in \mathscr{U}$. Then, for each $A \in \alpha$, there is $V \in \mathscr{U}$ such that $V A=A$. Since, in particular, $(\alpha, \beta) \in V^{\prime}$, it follows that $V A \in \beta$, and thus $\alpha \subset \beta$. By similar reasoning, $\beta \subset \alpha$, and so $\alpha=\beta$, and $(P, \mathscr{V})$ is Hausdorff.

Proposition 7. If $(P, \mathscr{V})$ is Hausdorff, then $(S, \mathscr{U})$ is Hausdorff.

Proof. Assume that $(S, \mathscr{U})$ is not Hausdorff. Then there exist distinct elements $y$ and $z$ in $S$ such that $(z, y) \in U$ for each $U \in \mathscr{U}$. Moreover, since $(S, \mathscr{U})$ is nontrivial, there is $x$ in $S, x \neq y$, and $V \in \mathscr{U}$ such that $(x, y) \notin V$. Let $W=S \times S-$ $\{(x, y)\}$. Since $W \supset V, W \in \mathscr{U}$. Let $\alpha=\langle W\rangle$, and $\beta=l$. Then $U W=S \times S$ for each $U \in \mathscr{U}$, and hence $(\alpha, \beta) \in U^{\prime}$ for each $U \in \mathscr{U}$. This conclusion contradicts the assumption that $(P, \mathscr{V})$ is Hausdorff, and this contradiction completes the proof.

Proposition 8. Let $(S, \mathscr{U})$ be an infinite uniform space with the property that, for each $U \in \mathscr{U}$, there exists $V \in \mathscr{U}$ such that $V U=U$. Then $(S, \mathscr{U})$ is discrete.

Proof. We will first prove the existence of a one-to-one mapping $j$ of $S$ 
onto $S$ such that $j \cap W$ is the empty set for some $W \in \mathscr{U}$. Since $(S, \mathscr{U})$ is nontrivial, there is $W_{1} \in \mathscr{U}$ such that $W_{1}=S \times S-\{(a, b)\}$, where $a \neq b$. Choose $U \in \mathscr{U}$ such that $U W_{1}=W_{1}$. If $V=S \times S-\{(z, b): z \neq b\}$, then $V \supset U$ and hence $V \in \mathscr{U}$. Let $W_{2}=V \cap V^{-1}$. Partition $S$ into two sets $R$ and $Q$, where $R=\left\{r_{i}: i \in I\right\}, Q=\left\{q_{i}: i \in I\right\}$, and $I$ is an index set that defines a one-to-one correspondence between $R$ and $S$ such that $q_{i}$ corresponds to $r_{i}$. Then $j=$ $\left\{\left(r_{i}, q_{i}\right): i \in I\right\} \cup\left\{\left(q_{i}, r_{i}\right): i \in I\right\}$ is a one-to-one mapping of $S$ onto $S$. Assume that $b$ is in $Q$. If $U_{1}=S \times S-\left\{\left(b, r_{i}\right): i \in I\right\}$, then $U_{1} \supset W_{2}$, hence $U_{1} \in \mathscr{U}$. Choose $V_{1} \in \mathscr{U}$ such that $V_{1} U_{1}=U_{1}$. Then $\left(q_{i}, r_{i}\right) \notin V_{1}$ for every $i \in I$; thus if $W=V_{1} \cap V_{1}^{-1}, j \cap W$ is the empty set.

To complete the proof, let $U=\{(x, y): y \neq j(x)\}$; then $U \supset W$, and so $U \in \mathscr{U}$. Choose $V \in \mathscr{U}$ such that $V U=U$. Let $(x, y) \in V$ and $w=j^{-1}(y)$. Then $(w, z) \in U$ whenever $z \neq j(w)$. If $x \neq y$, then $(w, x) \in U$; this implies that $(w, y) \in$ $V U$. But $V U=U$ and $j(w)=y$; hence $(w, j(w)) \in U$, a contradiction. Thus $x=y$, and $V$ is the diagonal in $S \times S$.

THEOREM 6. The following statements about the scale $(P, \mathscr{V})$ of a non-trivial uniform space $(S, \mathscr{U})$ are equivalent.

(a) $(S, \mathscr{U})$ is discrete.

(b) $(P, \mathscr{V})$ is discrete.

(c) $(P, \mathscr{V})$ is Hausdorff.

(d) For each $U \in \mathscr{U}$, there is $V \in \mathscr{U}$ such that $V U=U$.

(e) The uniform topology of $(P, \mathscr{V})$ is discrete.

(f) $(P, \mathscr{V})$ is totally disconnected.

Proof. If the diagonal of $S \times S$ is in $\mathscr{U}$, then clearly the diagonal of $P \times P$ is in $\mathscr{V}$, and so (a) implies (b), (b) obviously implies (c), and (c) implies (d) by Proposition 6. If $(S, \mathscr{U})$ is infinite, then (d) implies (a) by Proposition 8 . If $(S, \mathscr{U})$ is finite, then Propositions 6 and 7 imply that $(S, \mathscr{U})$ is Hausdorff, which is equivalent to discreteness for finite spaces; thus the first four statements are all equivalent. Also note that (b) implies (e) and (e) implies (c). Finally, (b) implies (f), and (f) implies (c) since a totally disconnected space is always $T_{1}$.

For the retracted scale, discreteness can be characterized as follows; we omit the proof.

THEOREM 7. The following statements are equivalent.

(a) $(S, \mathscr{U})$ is generated by a single entourage.

(b) The R-equivalence classes in the scale are open sets.

(c) The retracted scale uniform space is discrete.

It can also be shown that the retracted scale is totally disconnected whenever 
$(S, \mathscr{U})$ has a uniform basis consisting of entourages which are equivalence relations on $S$. Thus it is possible for the retracted scale to be totally disconnected but not discrete.

\section{References}

[1] D. Bushaw, 'On Boundedness in Uniform Spaces', Fund. Math. 56 (1965), 295-300.

[2] D. Bushaw, 'A Stability Criterion For General Systems', Math. Systems Theory, 1 (1967), $79-88$.

[3] D. Bushaw, 'The Scale of a Uniform Space', Proc. of the International Symposium on Topology and its Applications, Hercegnovi, Yugoslavia, August 25-31, 1968.

[4] D. C. Kent, 'On the Scale of a Uniform Space', Inventiones Math. 4 (1967), 159-164.

[5] S. Mrówka and W. J. Pervin, 'On Uniform Connectedness', Proc. Amer. Math. Soc. 15 (1964), 446-449.

Idaho State University

Pocatello, Idaho, USA

and

Washington State University

Pullman, Washington, USA 